\title{
Impact of COVID-19 Pandemic on Cancer Screening in the United States
}

\section{Sonikpreet Aulakh, MBBS MD ${ }^{1}$ \& Asher Chanan-Khan, MD $^{2}$}

How to cite: S Aulakh, A Chanan-Khan. Impact of covid-19 pandemic on cancer screening in the United States. Esculapio 2021;17(01):3-4

\section{DOI: https://doi.org/10.51273/esc21.2517123}

COVID-19 pandemic has exposed vulnerabilities all across the global healthcare systems including those within the United States. A systematic evaluation of these soft spots has been crucial in order to reengineer the healthcare system for enhanced competences and superior quality of care. One area that has been undoubtedly affected is the diagnosis and management of neoplastic diseases.

The healthcare system in the US witnessed an instantaneous implementation of a "social distancing" strategy, which was implemented in an effort to flatten the infectivity "curve". This required an urgent modification in the general administration of healthcare delivery, independent of COVID-19 infection status of a patient. For the non-COVID patients, it meant a shift from in-person to a virtual administration platform." (Royce et al., 2020) Neither the healthcare providers, nor the patients, or the hospital management were adequately prepared for this sudden transition. Various healthcare services offered through these healthcare systems were required to be triaged based upon patients' assessment of needs into either emergent, urgent or routine/non-urgent. Patients seeking services that fell in the non-urgent/routine clinical visits were encouraged to stay home until the pandemic simmered down/resolved. This strategy was erroneously predicated on a rather short anticipated duration of the pandemic. As expected, cancer screening visits were deemed non-urgent and thus most healthcare facilities in and outside the US

\footnotetext{
1 Sonikpreet Aulakh

2. Asher Chanan-Khan Departments of Medicine and Neurosciences, West Virginia University, WV 2. Department of Medicine, Cancer Biology and Immunology, Mayo
Clinic, FL.
}

Corrosponding Author:

Sonikpreet Aulakh, Departments of Medicine and Neurosciences, West

Virginia University, WV 26505sonikpreet.aulakh@hsc.wvu.edu suspended these services, inadvertently compromising the timely diagnosis of neoplastic disorders.

Over the last 50 years, we have witnessed major improvement in survival outcomes for all cancers collectively. (Siegel et al., 2020) A significant contributions to this feat is credited to the wellestablished cancer screening protocols allowing early detection, timely intervention and curtailing cancer related morbidity and mortality. (Duffy et al., 2020) Published data validates that cancer screening directly translates into lives saved. (Duffy \& Field, 2020) While screening algorithms are not available for all types of cancers, the Big Five most prevalent cancers (breast, prostate, colorectal, cervical and lung) have well defined and established screening protocols which are typically administered through primary care or cancer care centers. Despite benefit of screening, challenges have persisted in achieving maximal (or desirable) compliance by patients who are eligible for specific cancers. For instance, in the US, screening mammography is achieved in $74 \%$ of eligible women covered by commercial plans, 70\% by Medicare and only $59 \%$ by Medicaid. Data on uninsured women is expected to be even worse.(Peek $\&$ Han, 2004) Among the many reasons (socioeconomic status, health insurance, health education etc.) (Pruitt et al., 2009) an underappreciated factor is the psychological limitation in cancer screening. This factor has become far more relevant in context with the COVID-19 crisis.

The psychological limitation of cancer screening pertains to a sense of wellness among healthy individuals that foster complacency towards deferring timely need of cancer screening as established and proven through rigorous clinical 
investigations. Appropriately so, the healthcare system also equates cancer screening visits as "well visits". They are deemed non-urgent and planned visits for which the system relies upon patients' conviction to health maintenance. As often there is no perceived imminent danger to health, patients and healthcare providers' perception of these visits is that of an elective non-urgent nature. It is therefore not surprising that patients themselves, and the healthcare teams ascribed a low priority to cancer screening during the COVID-19 pandemic. This not only allowed much needed healthcare resources to be diverted to those actually fighting the COVID-19 infections, but also prevented unnecessary risk of exposure to those individuals who were not in urgent need of medical evaluation. Given this psyche, a nationwide decrease in cancer screening visits was recorded during the COVID-19 crisis. (Cancino et al., 2020) (Van Haren et al., 2020)

Another reason that directly impacted cancer screening visits, is the perceived risk of contracting COVID-19 when visiting a healthcare facility. Are all healthcare facilities truly high-risk environment for COVID-19? The drastic disengagement of preventative services (such as that for cancer) in the wake of COVID-19 crisis undoubtedly communicated this message to the masses. Can a more informed strategy been developed to minimize the impact of COVID-19 on cancer screening? For example, in high density areas of COVID-19 (such as NYC, Chicago or Los Angeles) transient complete cessation of cancer screening programs may be reasonable. Thereby extending accommodation to healthcare facilities with a high COVID burden to dispense resources towards acutely ill patients, protect staff and non-acute patients from infectivity risk. However, in cities with low COVID density, opportunities could still be leveraged cautiously to continue to offer these preventative services through employment of innovative strategies such as Telehealth. In fact, it is now increasingly recognized that ambulatory care and stand-alone diagnostic testing facilities (with appropriate PPE) pose comparably far less risk of COVID-19 infectivity vs. acute care hospital settings.

In reality, as the COVID-19 threat rapidly became a realization in the US (February 2020), the utilization of cancer screening procedures (colonoscopy, mammography, CT scans, pap smears etc.) were observed to significantly decline. Overall, nationally in the US a $60-80 \%$ decrease in cancer screening was recorded. (Cancino et al., 2020) (Van Haren et al., 2020) One study, evaluating claims data reported $87 \%$ reduction in mammograms, $83 \%$ reduction in pap smears and a $90 \%$ decrease in colonoscopies by early April 2020. (Mitchell, 2020) This delay in screening is undoubtedly going to have a major impact in the overall effect on cancer morbidity and mortality. In fact, the overall effects of delays in cancer screening nationally are already started to surface. It is estimated that over 22 million Americans will be compromised if testing for cancer screening remain at the low levels observed at the height of pandemic. This means that approximately 80,000 positive cancer cases can be expected to have a delay in their diagnosis. (Yong et al., 2020) (Bakouny et al., 2020) Sadly, delayed diagnosis of cancer in some of these cases may mean a lost opportunity for cure, while for many others it will result in upstaging of cancer and higher morbidity.(Giesen et al., 2020)(Pathania et al., 2021) Thus, COVID-19 is not only directly lethal to the patients, but also has the potential to indirectly amplify its mortal impact through suspension of the cancer screening services. As the US cautiously disengage from "social distancing", healthcare services are also testing waters to ramp up services for their patients. Thus, a steady recovery in cancer screening practices is fortunately anticipated. There remains significant ambiguity as to what is the best practice for reinstitution of these programs in the post-COVID era, which has yet to realize. However, it is clear that as the duration of this pandemic has extended significantly beyond the initial reported assessments, the need to reinstitute cancer screening programs to at least the pre-COVID momentum is an undeniable priority. 